\title{
Height structure of X-ray, EUV, and white-light emission in a solar flare ${ }^{\star}$
}

\author{
M. Battaglia and E. P. Kontar
}

\author{
School of Physics and Astronomy, University of Glasgow, Glasgow G12 8QQ, UK \\ e-mail: marina.battaglia@glasgow.ac.uk
}

Received 30 June 2011 / Accepted 22 July 2011

\begin{abstract}
Context. The bulk of solar flare emission originates from very compact sources located in the lower solar atmosphere and observable at a broad range of wavelengths such as near optical, UV, EUV, soft and hard X-rays, and gamma-rays. Nevertheless, very few spatially resolved imaging observations have been performed to determine the structure of these compact regions.

Aims. We investigate the above-the-photosphere heights of hard X-ray (HXR), EUV, and white-light (6173 $\AA$ ) continuum sources in the low atmosphere and the corresponding densities at these heights. By considering the collisional transport of solar energetic electrons, we also determine where and how much energy is deposited and compare these values with the emissions observed in HXR, EUV, and the continuum.

Methods. Simultaneous EUV/continuum images from AIA/HMI on-board SDO and HXR RHESSI images are compared to study a well-observed gamma-ray limb flare. Using RHESSI X-ray visibilities, we determine the height of the HXR sources as a function of energy above the photosphere. Co-aligning AIA/SDO and HMI/SDO images with RHESSI, we infer, for the first time, the heights and characteristic densities of HXR, EUV, and continuum (white-light) sources in the flaring footpoint of the magnetic loop.

Results. We find 35-100 keV HXR sources at heights of between 1.7 and $0.8 \mathrm{Mm}$ above the photosphere, below the $6173 \AA$ continuum emission that appears at heights 1.5-3 Mm and the peak of EUV emission originating near $3 \mathrm{Mm}$.

Conclusions. The EUV emission locations are consistent with energy deposition from low energy electrons of $\sim 12 \mathrm{keV}$ occurring in the top layers of the fully ionized chromosphere/low corona and not by $\gtrsim 20 \mathrm{keV}$ electrons that produce HXR footpoints in the lower neutral chromosphere. The maximum of white-light continuum emission appears between the HXR and EUV emission, presumably in the transition between ionized and neutral atmospheres, implying that it consists of free-bound and free-free continuum emission. We note that the energy deposited by low energy electrons is sufficient to explain the energetics of both the optical and UV emissions.
\end{abstract}

Key words. Sun: flares - Sun: X-rays, gamma-rays - Sun: radio radiation - Sun: UV radiation - acceleration of particles

\section{Introduction}

Solar flare emission is now observed over virtually the whole electromagnetic spectrum from radio frequencies as low as 10-100 MHz up to photon energies as high as a few hundred $\mathrm{MeV}$. The bulk of this emission originates from the chromosphere, a small part of the solar atmosphere that is narrow in height and much smaller than the size of a flaring region. Being only about $<3 \mathrm{Mm}\left(<4^{\prime \prime}\right)$ across, the study of the chromosphere is a formidable observational challenge in terms of both angular and temporal resolution especially for transient phenomena such as solar flares. During solar flares, large numbers of energetic electrons are accelerated in the solar corona and effectively release their energy into the dense chromosphere. These energetic particles are believed to be responsible (although not always directly) for the HXR, EUV, continuum "white-light" (WL), infrared, and radio emissions. On the basis of temporal correlation, the interconnection between these emissions was realized at an early stage (Najita \& Orrall 1970; Švestka 1970). Owing to the lack of height-resolving observations, spectroscopic data have mostly been used to identify the structure of the emitting region (e.g. Canfield 1974). Therefore, the actual

$\star$ Two movies are available in electronic form at http://www . aanda.org mechanisms of WL emission and their relation to HXR emitting electrons remains unclear (Neidig 1989; Neidig et al. 1993; Sylwester \& Sylwester 2000; Matthews et al. 2003; Potts et al. 2010; Watanabe et al. 2010; Kretzschmar 2011). It is not even clear whether the emission is optically thick or thin or both. Thus the characteristic heights of continuum WL emission in various models are between the photosphere and the upper chromosphere. Observations supporting both (Fang \& Ding 1995; $\mathrm{Xu}$ et al. 2006) a photospheric origin (e.g. Boyer et al. 1985; Ding et al. 1999; Chen \& Ding 2006) presumably due to radiative back-warming, and the generation of WL emission in the higher temperature regions of the upper chromosphere (e.g. Machado \& Rust 1974; Hudson 1972) have been made. The major observational challenge here is to have simultaneous HXR, UV, and WL images with sufficiently high angular resolution. The relative horizontal positions of HXR and WL flares have been investigated in detail and, although they have generally been found to agree (e.g. Krucker et al. 2011; Fletcher et al. 2007; Hudson et al. 2006; Metcalf et al. 2003), the uncertainty in the relative positions was large because of the limited absolute pointing accuracy of partial solar disk observations such as those from TRACE. Thus it was impossible to quantify the relative height of the sources above the photosphere in previous studies. With RHESSI (Lin et al. 2002) data, the height structure of 


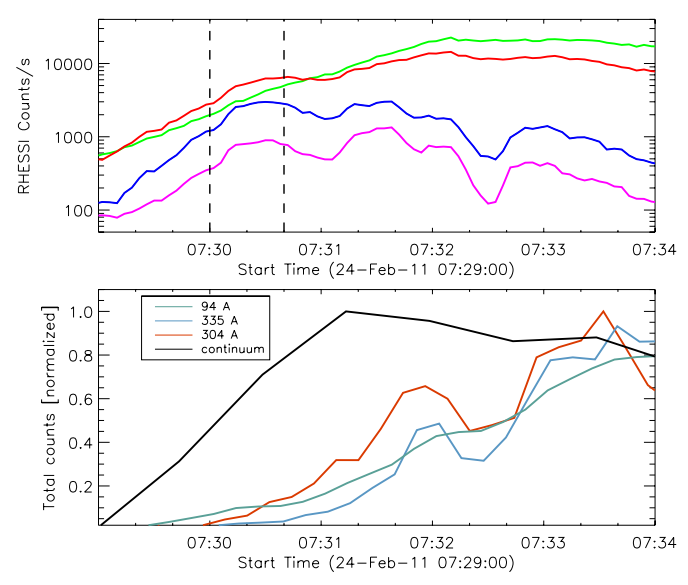

Fig. 1. Top: RHESSI lightcurves of the main flare phase in different energy bands: 6-12 keV (green), 12-25 keV (red), 25-50 keV (blue), 50-100 keV (purple). Bottom: normalized AIA and HMI emission emanating from location of the southern HXR footpoint as function of time at several wavelengths (see legend).

HXR sources has become accessible for detailed observational studies (Aschwanden et al. 2002; Kontar et al. 2008; Kontar \& Jeffrey 2010; Battaglia \& Kontar 2011; Saint-Hilaire et al. 2010). These observations have demonstrated that the higher energy HXR footpoints originate from progressively lower heights in the chromosphere, in good agreement with collisional downward propagation of energetic electrons (see Brown et al. 2002, for details). The newly available SDO continuum images from HMI (Wachter et al. 2011) and AIA EUV (Lemen et al. 2011) observations allow us to scrutinize the spatial structure and more importantly the height structure of various types of emission associated with HXR footpoints. In this paper, we present the first spatially resolved simultaneous observations of HXR, EUV, and WL emission from a footpoint of a solar limb flare observed with RHESSI, SDO/AIA, and HMI. Using the recently developed hard X-ray visibility analysis technique (Hurford et al. 2002; Schmahl et al. 2007), we determine the characteristic heights of the different types of emission using the method presented by (Battaglia \& Kontar 2011; Kontar et al. 2010, 2008). The results are consistent with the EUV and continuum WL being produced above the HXR footpoints by lower energy electrons $(\sim 12 \mathrm{keV})$, hence suggest not a photospheric but an upper chromospheric origin of WL emission.

\section{Height and density measurements}

The GOES M3.5 limb flare occurred on 2011 February 24 with three well-pronounced main HXR (above $\sim 30 \mathrm{keV}$ ) peaks between 07:29 and 07:33 UT and an associated filament eruption to the south of the flaring site. Figure 1 (top) shows the RHESSI lightcurves in different energy bands of the thermal and nonthermal emission. The attenuator state was 1 during the main phase of the flare and the live-time was higher than $90 \%$ during the whole period of the observations, hence making the effects of pulse pile-up negligible. The flare appeared in the active region AR11163, was well observed in enhanced EUV emission visible in all AIA filters, and also displayed $6173 \AA$ continuum emission observed by HMI. The continuum emission (with a maximum enhancement of $\sim 15 \%$ above the photospheric background) delineated two footpoints and followed the X-ray time profile. Unfortunately, AIA images became saturated at most wavelengths before the soft X-ray peak (2nd HXR peak) of the
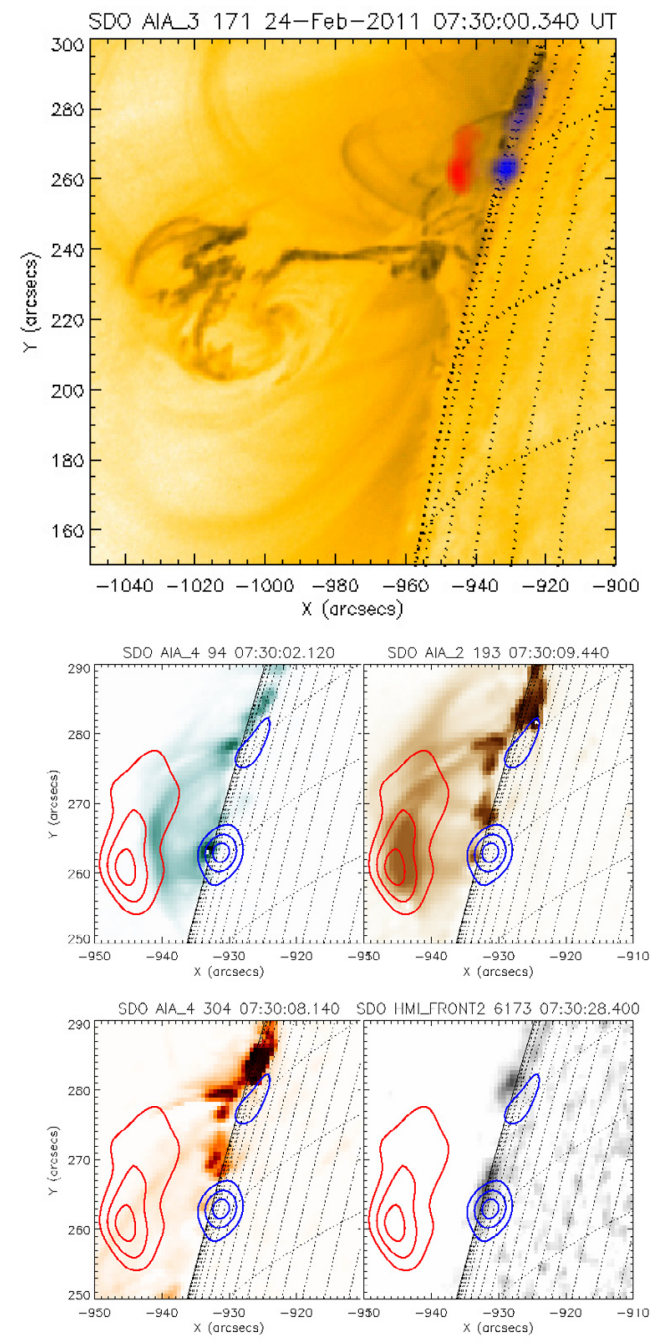

Fig. 2. Top: RHESSI soft (red) and hard X-ray emission (blue) superimposed on AIA $171 \AA$ image. Bottom: AIA difference images at three wavelengths and HMI image (bottom right). Contours at 50, 70 and $90 \%$ of the maximum emission in RHESSI CLEAN images between 07:30:00 and 07:30:40 UT at 6-12 keV (red) and 25-50 keV (blue) are overlaid. The temporal evolution is shown in the movies available in the on-line edition. RHESSI light-curves (cf. top panel of Fig. 1) were added to the animation of the bottom panel to provide a link between the total X-ray flux evolution and the corresponding images.

flare. Because of this saturation, we focus the analysis on the time of the first HXR peak. During the time around 07:30:10 UT, AIA was not saturated in the $94 \AA$, $193 \AA$, $304 \AA$, and $335 \AA$ filters near the location of the southern footpoint.

To study the flare related enhancement in the EUV and continuum emission, the image from one minute before the analyzed time was subtracted. Figure 2 displays difference images in AIA 94, 193 and $304 \AA$ wavelengths and the HMI continuum ${ }^{1}$. For the RHESSI analysis, a $40 \mathrm{~s}$ time interval at the first peak of the flare from 07:30:00 UT was chosen (see Fig. 1). The contours in Fig. 2 indicate the RHESSI emission between 07:30:00 and 07:30:40 UT at 50, 70, and 90\% of the maximum in CLEAN images (Hurford et al. 2002). The images show a coronal (SXR) source above the limb and two footpoint (HXR) sources (north and south). During the first peak, the southern footpoint source

\footnotetext{
1 Animated images of the flare are also available at http://www. astro.gla.ac.uk/users/mbattaglia/20110224_ online_material/
} 
was much more intense than the northern footpoint. Thus we focused the height analysis on the southern footpoint. We note that in the subsequent peaks the northern footpoint reached the same intensity as the southern footpoint. The RHESSI spectrum suggests emission well beyond $200 \mathrm{keV}$ into the $\gamma$-ray range. During the analyzed time interval, the HXR spectrum is well fitted with a thermal component and a single thick-target power law with spectral index $\delta=3.9$. The flare was also observed by Fermi/GBM (Meegan et al. 2009) and the corresponding spectrum from FERMI data was found to be virtually identical to the RHESSI spectrum. The time evolution of the EUV emission at the position of the southern HXR footpoint can be seen in Fig. 1 (bottom). The total flux in a region of 5 by 10 arcsec extent around the position of the southern HXR footpoint is shown.

Because of the limb location of the flare a height analysis is possible. However, SDO observations alone cannot infer the heights of the sources at different EUV wavelengths or for white-light emission. Here, utilizing RHESSI observations, we find the density structure of the chromosphere and importantly the reference height (projected radius of the point at the photospheric level beneath the HXR sources) as described by Battaglia $\&$ Kontar (2011) and Kontar et al. (2010, 2008). Following the method described by those authors, we use visibility forward fitting to find the position of the southern footpoint as a function of HXR energy in several energy bands (30-40 keV, 40-55 keV, $55-80 \mathrm{keV}, 80-130 \mathrm{keV})$. The energy bands were chosen to be large enough to guarantee good count statistics for reliable fits. Both the southern and the northern footpoints are fitted with circular Gaussians. This assures that the emission of the northern footpoint is properly accounted for, even if it is not intense enough to permit fit parameters to be determined with acceptable errors. The flare morphology was such that the radial direction (direction of electron propagation along the loop) can be seen along the $x$-direction in the images (see Fig. 2). An exponential density profile (hydrostatic atmosphere at lower heights), $n(h)=$ $n_{\mathrm{cs}}+n_{\mathrm{ph}} \exp \left(-\left(h-r_{\mathrm{ref}}\right) / h_{0}\right)$, was fitted to the positions found by visibility forward fitting, where $n_{\mathrm{cs}}=4.6 \times 10^{10} \mathrm{~cm}^{-3}$ is the (constant) loop density, $n_{\mathrm{ph}}=1.16 \times 10^{17} \mathrm{~cm}^{-3}$ the photospheric density (Vernazza et al. 1981), which corresponds to $\tau_{500 \mathrm{~nm}}=1$, and $h$ is the height above the photosphere ${ }^{2}$. The loop density $n_{\mathrm{cs}}$ was assumed to be the same as the coronal source density determined from the emission measure given by the spectral fit and the size of the coronal source in RHESSI 6-12 keV images. Fitting the HXR energy-position relation with the above density model, we find a scale height of $h_{0}=211 \pm 46 \mathrm{~km}$ and a reference height for the radial distance between the solar disk center and the point under the footpoints at the photosphere of $r_{\text {ref }}=929.4 \pm 0.3 \mathrm{arcsec}$. The photospheric reference distance $r_{\text {ref }}$ is used to find the absolute height of the EUV and WL sources above the photosphere. Using the HXR source size as the size of the magnetic flux tube, we selected a stripe along the $x$-direction of 5 arcsec width in the $y$-direction over the position of the southern RHESSI footpoint. At each horizontal distance $x$, the AIA and HMI counts were summed in the $y$-direction. The height of the emission is simply the $x$-position minus $r_{\text {ref }}$ as found above. This is shown in Fig. 3. Proper co-alignment of the images is crucial for this study. The RHESSI disk center is known to an accuracy higher than 0.2 arcsec. Using SDO full disk images, the

\footnotetext{
${ }^{2}$ In this work, we use a fit to the full expression for the HXR flux maximum and not the simplified version given by Eq. (7) of Brown et al. (2002). The latter is likely to underestimate the densities in a sharply changing chromosphere. See also Battaglia \& Kontar (2011) and Kontar et al. $(2010,2008)$.
}

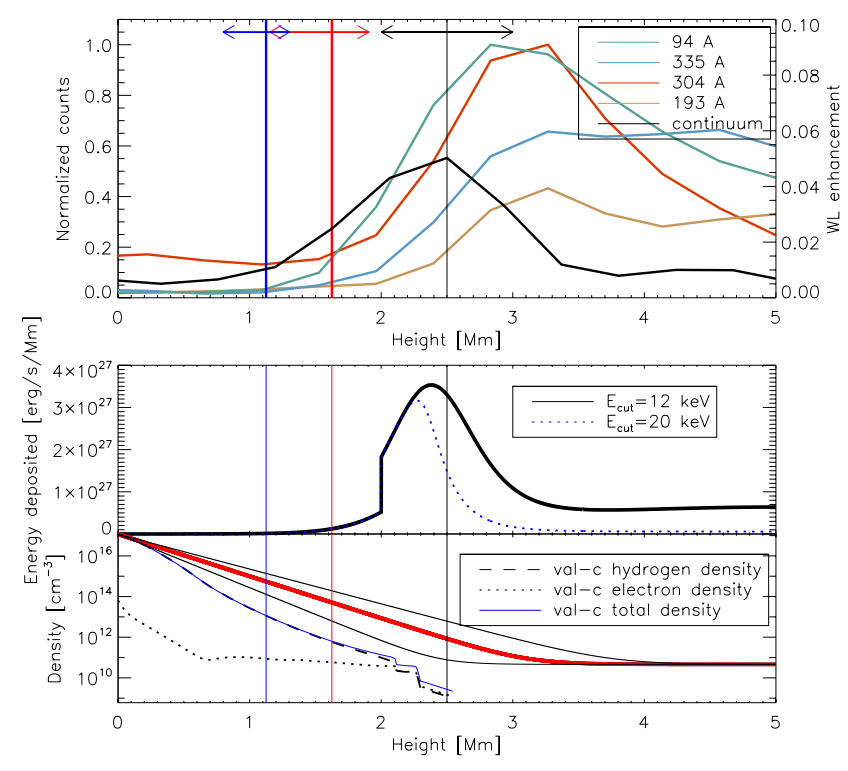

Fig. 3. Top: height of HXR emission at the lowest (red vertical lines) and highest (blue vertical lines) imaged energies, EUV emission at different wavelengths (see legend), and WL relative enhancement where the black line indicates the height of maximum enhancement. Arrows indicate the corresponding uncertainties. Middle: energy deposition rate as a function of height for two different cases of electron cutoff energy. Bottom: fitted density model (thick solid line). The thin solid lines give the lower and higher limits of the density determined by the uncertainty in the fit of the scale height. The model from Vernazza et al. (1981) is given for comparison.

disk center of AIA and HMI can be determined. This position of solar-disk center is found to deviate by only $-0.39 / 0.67$ arcsec in the $x / y$ direction from that of RHESSI, a deviation that, although small, was taken into account. The larger uncertainty is related to the roll-angle of the SDO images (rotation around the disk center). To investigate the effect, the HMI image was rotated by 0.1 degrees relative to the Sun center. This uncertainty translates mostly into a $y$-direction uncertainty of $<1.6$ arcsec and $<0.5$ for the $x$-direction for the limb event under study. For the height measurements of the EUV and HMI emissions relative to the RHESSI emission, we have taken this uncertainty into account as shown in Fig. 3. We find that the RHESSI sources at $35-100 \mathrm{keV}$ originate from heights of between 1.7 and $0.8 \mathrm{Mm}$. The WL emission peaks at $2.5 \mathrm{Mm}$, which is $\sim 1 \mathrm{Mm}$ above the $30 \mathrm{keV}$ HXR emission, suggesting it originates from the upper chromosphere.

Using the density model found above and a power-law electron injection rate $F_{0}\left(E_{0}\right)=F_{\text {norm }} E_{0}^{-\delta}$ (electrons s ${ }^{-1} \mathrm{keV}^{-1}$ ) found from the spatially integrated RHESSI X-ray spectrum, we can compute the energy deposition rate $\left(\operatorname{ergs~s}{ }^{-1} \mathrm{Mm}^{-1}\right)$ as a function of height (Brown 1973),

$\frac{\mathrm{d} U}{\mathrm{~d} h}=K\left[\Lambda_{\mathrm{e} H} n_{\mathrm{H}}(h)+\Lambda_{\mathrm{ee}} n_{\mathrm{e}}(h)\right] \int_{E} \frac{F(E, h)}{E} \mathrm{~d} E$,

where $F(E, h)$ is the electron rate spectrum (Brown 1971), $K=2 \pi e^{4}$ where $\Lambda_{\mathrm{eH}}$ and $\Lambda_{\mathrm{ee}}$ are the Coulomb logarithms for electron-neutral and electron-electron collisions, and $n_{\mathrm{H}}(h)$ and $n_{\mathrm{e}}(h)$ are the hydrogen and electron number densities respectively. The resulting energy deposition rate as a function of height given by Eq. (1) is shown in Fig. 3 where we have assumed a step change in ionization from fully ionized to neutral at a height of $2 \mathrm{Mm}$. As expected, the deposition rate depends on the low energy cut-off of the injected electron spectrum 
$F_{0}\left(E_{0}\right)$. From the thick target fit to the RHESSI spatially integrated HXR spectrum the cut-off energy is around $E_{\mathrm{c}}=12 \mathrm{keV}$ and the spectral index $\delta=3.9$. This leads to a total power of $\sim 2.7 \times 10^{27} \mathrm{erg} \mathrm{s}^{-1}$. However, the cut-off is generally unknown as the low energy part of the X-ray spectrum is dominated by thermal emission, so lower cut-off values are possible and likely (e.g. Emslie 2003; Hannah et al. 2009). However, even with $E_{\mathrm{c}}=12 \mathrm{keV}$, the energy deposited appears much larger than that emitted at other wavelengths. For the southern footpoint, an enhancement of $\sim 5 \%$ above the total solar irradiance of $3.4 \times 10^{26} \mathrm{erg} \mathrm{s}^{-1} \operatorname{arcsec}^{-2}$ over a source area of $\sim 20 \operatorname{arcsec}^{2}$ leads to a total power emitted of $3.4 \times 10^{26} \mathrm{erg} \mathrm{s}^{-1}$. The energy in the non-thermal electrons is therefore up to an order of magnitude larger than that needed to power the WL emission, which often constitutes the bulk of flare emitted energy (Neidig 1989). We note that the WL energetics obtained using the $6173 \AA$ continuum is found to be lower than at TRACE wavelengths near the $1700 \AA$ continuum, and assuming black body emission to deal with the TRACE response (e.g. Fletcher et al. 2007), but close to the values reported by Neidig (1989) based on spectroscopy above $2500 \AA$. Various optical bands indeed show different levels of enhancement during flares (Kretzschmar 2011). In addition, the maximum of $6173 \AA$ continuum is reached later in the flare, after the analyzed time interval.

\section{Discussion and conclusions}

Our observations suggest that the WL emission originates from either the upper chromosphere or lower transition region with a density of between $10^{11} \mathrm{~cm}^{-3}$ and $10^{13} \mathrm{~cm}^{-3}$. These upper and lower limits of the density are due to the uncertainties in the fit of the density scale height. Figure 3 (bottom) displays the fitted density model, as well as the upper and lower limits to the density. The arrows in Fig. 3 (top) illustrate the uncertainty in the RHESSI source heights resulting from the uncertainty in the density scale height. The peak of WL emission is found to be about $1 \mathrm{Mm}$ higher than the position of the lowest observed RHESSI energy range (30-40 keV). Despite all the uncertainties in the height caused by the density fit or the relative pointing error, the WL source is still above the 30-40 keV RHESSI sources. Owing to the presence of the SXR coronal emission in the X-ray spectrum, the non-thermal HXR spectrum below $\approx 20 \mathrm{keV}$ is not known. However, extrapolating the height function found in Sect. 2 to lower energies, a source at $12 \mathrm{keV}$ would be seen at about $2 \mathrm{Mm}$. This coincides with the height of the maximum energy deposition found from Eq. (1) for cut-off energies of $12 \mathrm{keV}$ (Fig. 2). The observed position of the maximum of the WL emission would then correspond to energy deposition by the low energy part of the non-thermal electron distribution, in contrast to the assumption often made that the cut-off in electron energies is of the order of $20 \mathrm{keV}$. This also suggests that WL and HXR sources above $30 \mathrm{keV}$ should be spatially separated by $<1 \mathrm{Mm}$ with decreasing separation in events towards the disk center. However, we note that the height of the maximum energy deposition, especially in the case of low initial cutoff energies, is influenced by the loop density and the length of the loop. Assuming that the acceleration of the particles happens at the center of what is observed as the coronal source, Fig. 2 indicates a precipitation distance of about 13 arcsec from the site of acceleration to the footpoints. This value was used for the computation of the energy deposition rate. In this case, the maximum energy deposition of electrons with energy $\lesssim 12 \mathrm{keV}$ will be near the top of the loop. Should the loop density be lower or the acceleration region extended, the lower energy electrons would deposit their energy at greater depths. The other interesting aspect of this flare is that HXR emission above $20 \mathrm{keV}$ is well fitted by a single power-law. Non-uniform ionization of plasma produces a break at the energy corresponding to the column depth of the transition region, which is observed in other flares (Kontar et al. 2002; Su et al. 2011). The absence of this break point to the transition region above the stopping depth of $20 \mathrm{keV}$ electrons is again consistent with the height measurements. The observation of strong emission from heights $\geq 2 \mathrm{Mm}$ suggests that a substantial part of the WL continuum is formed in an optically thin or finite optically thick region with ionized or partially ionized plasma in the upper chromosphere favoring free-bound and freefree emission of the $6173 \AA$ continuum. This is also supported by the height of the AIA emission at 94, 335, and $193 \AA$ (representing temperatures $>10^{6} \mathrm{~K}$ ) originating from around $3 \mathrm{Mm}$ and above the WL emission peak. Finally, it is likely that the WL is powered by lower energy electrons $\sim 12 \mathrm{keV}$, which do not penetrate deeply into the solar chromosphere.

Acknowledgements. The authors thank the anonymous referee for helpful comments and suggestions. This work is supported by the Leverhulme Trust (M.B., E.P.K.) and a STFC rolling grant (E.P.K.). Financial support by the European Commission through the FP7 HESPE network is gratefully acknowledged.

\section{References}

Aschwanden, M. J., Brown, J. C., \& Kontar, E. P. 2002, Sol. Phys., 210, 383 Battaglia, M., \& Kontar, E. P. 2011, ApJ, 735, 42

Boyer, R., Sotirovsky, P., Machado, M. E., \& Rust, D. M. 1985, Sol. Phys., 98, 255

Brown, J. C. 1971, Sol. Phys., 18, 489

Brown, J. C. 1973, Sol. Phys., 31, 143

Brown, J. C., Aschwanden, M. J., \& Kontar, E. P. 2002, Sol. Phys., 210, 373

Canfield, R. C. 1974, Sol. Phys., 34, 339

Chen, Q. R., \& Ding, M. D. 2006, ApJ, 641, 1217

Ding, M. D., Fang, C., \& Yun, H. S. 1999, ApJ, 512, 454

Emslie, A. G. 2003, ApJ, 595, L119

Fang, C., \& Ding, M. D. 1995, A\&AS, 110, 99

Fletcher, L., Hannah, I. G., Hudson, H. S., \& Metcalf, T. R. 2007, ApJ, 656, 1187

Hannah, I. G., Kontar, E. P., \& Sirenko, O. K. 2009, ApJ, 707, L45

Hudson, H. S. 1972, Sol. Phys., 24, 414

Hudson, H. S., Wolfson, C. J., \& Metcalf, T. R. 2006, Sol. Phys., 234, 79

Hurford, G. J., Schmahl, E. J., Schwartz, R. A., et al. 2002, Sol. Phys., 210, 61

Kontar, E. P., \& Jeffrey, N. L. S. 2010, A\&A, 513, L2

Kontar, E. P., Brown, J. C., \& McArthur, G. K. 2002, Sol. Phys., 210, 419

Kontar, E. P., Hannah, I. G., \& MacKinnon, A. L. 2008, A\&A, 489, L57

Kontar, E. P., Hannah, I. G., Jeffrey, N. L. S., \& Battaglia, M. 2010, ApJ, 717, 250

Kretzschmar, M. 2011, A\&A, 530, A84

Krucker, S., Hudson, H. S., Jeffrey, N. L. S., et al. 2011, ApJ, in press Lemen, J. R., Title, A. M., Akin, D. J., et al. 2011, Sol. Phys., in press Lin, R. P., Dennis, B. R., Hurford, G. J., et al. 2002, Sol. Phys., 210, 3 Machado, M. E., \& Rust, D. M. 1974, Sol. Phys., 38, 499

Matthews, S. A., van Driel-Gesztelyi, L., Hudson, H. S., \& Nitta, N. V. 2003, A\&A, 409, 1107

Meegan, C., Lichti, G., Bhat, P. N., et al. 2009, ApJ, 702, 791

Metcalf, T. R., Alexander, D., Hudson, H. S., \& Longcope, D. W. 2003, ApJ, 595,483

Najita, K., \& Orrall, F. Q. 1970, Sol. Phys., 15, 176

Neidig, D. F. 1989, Sol. Phys., 121, 261

Neidig, D. F., Wiborg, P. H., \& Gilliam, L. B. 1993, Sol. Phys., 144, 169

Potts, H., Hudson, H., Fletcher, L., \& Diver, D. 2010, ApJ, 722, 1514

Saint-Hilaire, P., Krucker, S., \& Lin, R. P. 2010, ApJ, 721, 1933

Schmahl, E. J., Pernak, R. L., Hurford, G. J., Lee, J., \& Bong, S. 2007, Sol. Phys., 240,241

Su, Y., Holman, G. D., \& Dennis, B. R. 2011, ApJ, 731, 106

Sylwester, B., \& Sylwester, J. 2000, Sol. Phys., 194, 305

Švestka, Z. 1970, Sol. Phys., 13, 471

Vernazza, J. E., Avrett, E. H., \& Loeser, R. 1981, ApJS, 45, 635

Wachter, R., Schou, J., Rabello-Soares, M. C., et al. 2011, Sol. Phys., 19

Watanabe, K., Krucker, S., Hudson, H., et al. 2010, ApJ, 715, 651

Xu, Y., Cao, W., Liu, C., et al. 2006, ApJ, 641, 1210 